\title{
High technology by CZT cameras: It is time to join forces
}

\author{
Adriana D'Antonio, $\mathrm{MD},{ }^{\mathrm{a}}$ Roberta Assante, $\mathrm{MD}, \mathrm{PhD},{ }^{\mathrm{a}}$ \\ Emilia Zampella, MD, PhD, and Wanda Acampa, $M D, P^{a}$ \\ a Department of Advanced Biomedical Sciences, University Federico II, Naples, Italy
}

Received Aug 5, 2021; accepted Aug 5, 2021

doi: 10.1007/s12350-021-02777-6

See related article, pp. 2311-2321

Single-photon emission-computed tomography (SPECT) myocardial perfusion imaging (MPI) is a standard and valuable tool for diagnosis and risk stratification of patients with suspected or known coronary artery disease (CAD), traditionally based on visual and semiquantitative assessment of severity and extent of perfusion abnormalities. ${ }^{1}$ Although the standard semiquantitative approach remains a sensitive means for diagnosing or ruling out the presence of obstructive CAD, it often reveals only the coronary territory subtended by the vessel with the most severely flowlimiting stenosis, underestimating the extent of disease in patients with multivessel disease. This limitation is due to diffuse abnormal coronary vasodilator reserve in patients with extensive $\mathrm{CAD}$, leading to the so-called balanced reduction in myocardial perfusion, reduced flow heterogeneity, and underestimation of the extent of underlying obstructive CAD. ${ }^{2}$ Myocardial flow reserve (MFR), obtained by the ratio between hyperemic and baseline myocardial blood flow (MBF), is a well-validated index for the assessment of blood flow impairment, integrating the hemodynamic effects of epicardial coronary stenosis, diffuse atherosclerosis, and microvascular dysfunction, which may enhance prognostic assessment and risk stratification in different categories of patients including those with risk factors such as diabetes mellitus and resistant hypertension. ${ }^{3-6}$ Although MBF quantification can be performed with

Reprint requests: Wanda Acampa, MD, PhD, Department of Advanced Biomedical Sciences, University Federico II, Naples, Italy; acampa@unina.it

J Nucl Cardiol 2022;29:2322-4.

$1071-3581 / \$ 34.00$

Copyright (c) 2021 American Society of Nuclear Cardiology. different techniques, including cardiac magnetic resonance and cardiac-computed tomography angiography (CCTA), positron emission tomography (PET) has emerged as a reliable and well-validated modality with established diagnostic and prognostic value in the context of coronary artery disease (CAD) and nonischemic heart disease. ${ }^{7-9}$ Several PET tracers are available for MBF and MFR quantification, including ${ }^{15} \mathrm{O}$-water, ${ }^{13} \mathrm{~N}$ ammonia, and ${ }^{82} \mathrm{Rb}$; however, their diffuse application in the clinical scenario is limited by technical complexity and elevated operative costs. Recently, novel cardiac-dedicated gamma cameras with semiconductor cadmium-zinc-telluride (CZT) detectors have been introduced in the clinical practice for cardiac imaging, improving sensitivity, and image resolution and dramatically reducing the imaging time and radiation dose while maintaining high diagnostic accuracy as compared with conventional cameras. ${ }^{10,11}$ These new cameras allow to perform dynamic SPECT MPI by list-mode acquisition, adding to the standard perfusion evaluation the possibility to estimate MBF and MFR. Previous studies investigated the feasibility of MBF and MFR quantification by CZT-SPECT imaging, in patients with suspected or known CAD. ${ }^{12-14}$ Moreover, it has been demonstrated a good correlation between CZT-SPECT and PET for both MBF and MFR quantification. ${ }^{15,16}$

In the current issue of Journal of Nuclear Cardiology, Liu et al. ${ }^{17}$ aimed to evaluate the feasibility of MBF and MFR absolute quantification, using a cardiac-dedicated ultrafast CZT camera (D-SPECT, Spectrum Dynamics, Caesarea, Israel). The authors estimated the added value of global and regional MBF and MFR to static perfusion and ECG-gated SPECT parameters in patients with left main or multivessel CAD, with respect to invasive coronary angiography and CCTA data. For this purpose, the authors retrospectively analyzed data of 32 patients with suspected CAD and negative MPI (SSS $\leq 4$ ), who underwent a one-day rest/stress dynamic 99m-Technetium CZT-SPECT MPI and a subsequent 
coronary angiography or CCTA within 6 months from MPI, according to which they were divided into three groups: 10 patients with nonsignificant stenosis, 10 patients with one-vessel disease, and 12 patients with multivessel disease. The acquisition protocol was modified according to the WATERDAY study, and regadenoson was replaced by dipyridamole for stress test. $^{15,18}$ The authors found that global hyperemic MBF was lower in patients with CAD associated with a significantly higher SSS in multivessel disease patients as compared to patient without CAD. However, there were no differences in MFR between the groups. Similarly, at per-vessels analysis, regional hyperemic and baseline MBF were significantly lower in stenotic vessels, whereas MFR and regional perfusion data did not show significant differences, as compared to normal vessels.

Previous reports investigated the relationship between anatomical disease and quantitative perfusion measurements by CZT-SPECT, providing different results. ${ }^{1-13}$. In particular, in a recent report, ${ }^{14}$ it has been demonstrated that both hyperemic MBF and MFR resulted significantly lower in patients with obstructive CAD as compared to those without, in both per patients and vessels analysis.

Differently, Liu et al. ${ }^{17}$ found that global hyperemic MBF resulted to be superior to global MFR and other MPI parameters in detecting multivessel CAD, with an optima cut-off value of $3.5 \mathrm{ml} / \mathrm{g} / \mathrm{min}$. Similarly, regional MBFs resulted more accurate than regional MFR in detecting vessel stenosis and predicting both anatomical stenosis and functional severity on a per-vessels analysis. This could be explained considering that, although there was no sex-specific difference in regional hyperemic MBF, higher baseline MBF, and subsequent lower MFR were noted in women with 1-vessel disease along with higher prevalence of small heart that could affect perfusion and dynamic flow data and may contribute to the non-significance of regional MFR to predict vessel stenosis. However, this point highlights the necessity for more studies to be performed on a much larger population considering racial and gender factors.

Different MFR and hyperemic MBF cut-off values have been previously proposed for the identification of obstructive CAD by CZT-SPECT imaging. ${ }^{11-15}$ In the WATERDAY study, the authors found an optimal cutoff of $2.5 \mathrm{ml} / \mathrm{min} / \mathrm{g}$ for global stress MBF and 2.1 for MFR in detecting obstructive CAD. ${ }^{15}$ Similarly, in a population of 173 patients with suspected or known CAD, a MFR value of 2.6 was identified as the best trade-off between sensitivity and specificity for identifying obstructive CAD. ${ }^{14}$ In the present study, Liu et al. ${ }^{17}$ find out higher optimal cut-off values for both global and regional hyperemic MBF in predicting multivessel CAD. This discrepancy may be partially explained considering that patients with multivessel CAD included in the current study showed a higher prevalence of previous coronary interventions, and an improvement in coronary hyperemic blood flow has been demonstrated after a coronary revascularization procedures. ${ }^{19}$

Another point to emphasize is that, although the optimal cut-off values for MBF and MFR were validated using regadenoson as stress agent, ${ }^{15}$ several studies observed that dipyridamole, regadenoson, and adenosine can induce an equivalent level of hyperemia and similar performance in the detection of CAD. ${ }^{14,18,20}$ In the current study, Liu et al. ${ }^{17}$ confirmed the feasibility of dipyridamole as stress agent finding a positive correlation between the change of heart rates from rest to stress state and the global MFR as well as $\triangle \mathrm{MBF}$.

Although previous published data confirmed a relationship between myocardial perfusion defect and MFR in patients with $\mathrm{CAD},{ }^{12-14}$, the significance of reduced MBF and MFR in a territory with normal perfusion remains to be investigated.

In a recent report, ${ }^{21}$ hyperemic $\mathrm{MBF}$ and MFR obtained by ${ }^{82} \mathrm{Rb}$-PET resulted to be lower in diabetic patients with normal MPI as compared to nondiabetic patients. Differently, baseline $\mathrm{MBF}$ was comparable among patients with and without diabetes. In the same report, the presence of diabetes in patients with reduced MFR was associated with higher rate of cardiac events during follow-up.

In the current study, ${ }^{17}$ a higher prevalence of diabetes mellitus and smoking history was found in patient with $\mathrm{CAD}$ as compared to those without, suggesting that quantification of MBF and MFR could play a critical role in diagnosis and identification of high-risk patients. In addition, the authors provide a diagnostic algorithm, according to the recent ESC guidelines, ${ }^{22}$ for patients with negative MPI (SSS $\leq 4)$ and moderate to high clinical likelihood for multivessel CAD and balanced ischemia, using a clinically available method to quantitatively measure MBF and MFR using dynamic ${ }^{99 \mathrm{~m}} \mathrm{Tc}-$ perfusion SPECT with a CZT camera.

The present report by Liu et al. ${ }^{17}$ is a single center and observational study involving a limited number of patients, such as the majority of published data. The diagnosis of multivessel disease may be one of the most appropriate indications for the use of dynamic CZT imaging in clinical practice; however, several questions remain still unanswered such as the identification of optimal MBF and MFR cut-off values and their further validation.

We, as users, could improve such as promising technology; if we join the forces, diagnostic and prognostic applications of quantitative CZT-SPECT imaging 
will be better defined in larger simple size multicenter and randomized studies.

\section{Declarations}

The authors declare that they have no conflict of interest.

\section{References}

1. Iskandrian AE, Hage FG, Shaw LJ, Mahmarian JJ, Berman DS (2014) Serial myocardial perfusion imaging: Defining a significant change and targeting management decisions. JACC Cardiovasc Imaging 7:79-96

2. Berman DS, Kang X, Slomka PJ, Gerlach J, de Yang L, Hayes SW et al (2007) Underestimation of extent of ischemia by gated SPECT myocardial perfusion imaging in patients with left main coronary artery disease. J. Nucl. Cardiol. 14:521-28

3. Assante R, Zampella E, Arumugam P, Acampa W, Imbriaco M, Tout D et al (2017) Quantitative relationship between coronary artery calcium and myocardial blood flow by hybrid rubidium- 82 PET/ CT imaging in patients with suspected coronary artery disease. J. Nucl. Cardiol. 24:494-501

4. Gaudieri V, Acampa W, Rozza F, Nappi C, Zampella E, Assante R. et al. Coronary vascular function in patients with resistant hypertension and normal myocardial perfusion: A propensity score analysis. Eur. Heart J. Cardiovasc. Imaging. 2019.

5. Assante R, Acampa W, Zampella E, Arumugam P, Nappi C, Gaudieri V. et al. Coronary atherosclerotic burden vs. coronary vascular function in diabetic and nondiabetic patients with normal myocardial perfusion: A propensity score analysis. Eur. J. Nucl. Med. Mol. Imaging 2017;44:1129-35.

6. Daniele S, Nappi C, Acampa W, Storto G, Pellegrino T, Ricci F et al (2011) Incremental prognostic value of coronary flow reserve assessed with single-photon emission computed tomography. $J$. Nucl. Cardiol. 18:612-19

7. Zampella E, Acampa W, Assante R et al (2018) Combined evaluation of regional coronary artery calcium and myocardial perfusion by ${ }^{82} \mathrm{Rb}$ PET/CT in the identification of obstructive coronary artery disease. Eur. J. Nucl. Med. Mol. Imaging 45:52129

8. Ziadi MC, Dekemp RA, Williams K, Guo A, Renaud JM, Chow BJ et al (2012) Does quantification of myocardial flow reserve using rubidium-82 positron emission tomography facilitate detection of multivessel coronary artery disease? J. Nucl. Cardiol. 19:670-80

9. Fiechter M, Ghadri JR, Gebhard C, Fuchs TA, Pazhenkottil AP, Nkoulou RN et al (2012) Diagnostic value of $13 \mathrm{~N}$-ammonia myocardial perfusion PET: Added value of myocardial flow reserve. J. Nucl. Med. 53:1230-34

10. Acampa W, Buechel RR, Gimelli A (2016) Low dose in nuclear cardiology: State of the art in the era of new cadmium-zinctelluride cameras. Eur. Heart J. Cardiovasc. Imaging 17:591-95

11. Mannarino T, Assante R, Ricciardi C, Zampella E, Nappi C, Gaudieri V et al (2021) Head-to-head comparison of diagnostic accuracy of stress-only myocardial perfusion imaging with conventional and cadmium-zinc telluride single-photon emission computed tomography in women with suspected coronary artery disease. J. Nucl. Cardiol. 28:888-97
12. Ben-Haim S, Murthy VL, Breault C, Allie R, Sitek A, Roth N et al (2013) Quantification of myocardial perfusion reserve using dynamic SPECT imaging in humans: A feasibility study. J. Nucl. Med. 54:873-79

13. Ben Bouallegue F, Roubille F, Lattuca B, Cung TT, Macia JC, Gervasoni R et al (2015) SPECT Myocardial Perfusion Reserve in Patients with Multivessel Coronary Disease: Correlation with Angiographic Findings and Invasive Fractional Flow Reserve Measurements. J. Nucl. Med. 56:1712-17

14. Acampa W, Assante R, Mannarino T, Zampella E, D'Antonio A, Buongiorno P, Gaudieri V, Nappi C, Giordano A, Mainolfi CG, Petretta M, Cuocolo A (2020) Low-dose dynamic myocardial perfusion imaging by CZT-SPECT in the identification of obstructive coronary artery disease. Eur. J. Nucl. Med. Mol. Imaging 47:1705-12

15. Agostini D, Roule V, Nganoa C, Roth N, Baavour R, Parienti JJ et al. First validation of myocardial flow reserve assessed by dynamic (99m)Tc-sestamibi CZT-SPECT camera: head to head comparison with (15)O-water PET and fractional flow reserve in patients with suspected coronary artery disease. The WATERDAY study. Eur. J. Nucl. Med. Mol. Imaging 2018;45:1079-90

16. Acampa W, Zampella E, Assante R, Genova A, De Simini G, Mannarino T, D'Antonio A et al (2020) Quantification of myocardial perfusion reserve by CZT-SPECT: A head to head comparison with ${ }^{82}$ Rubidium PET imaging. J. Nucl. Cardiol. h ttps://doi.org/10.1007/s12350-020-02129-w

17. Liu FS, Wang SY, Shiau YC, Wu YW. Integration of quantitative absolute myocardial blood flow estimates from dynamic CZTSPECT improves the detection of coronary artery disease. J. Nucl. Cardiol. 2021

18. Brana Q, Thibault F, Courtehoux M, Metrard G, Ribeiro MJ, Angoulvant D et al (2020) Regadenoson versus dipyridamole: Evaluation of stress myocardial blood flow response on a CZTSPECT camera. J. Nucl. Cardiol. https://doi.org/10.1007/s12350020-02271-5

19. Nijjer SS, Petraco R, van de Hoef TP, Sen S, van Lavieren MA, Foale RA et al. Change in coronary blood flow after percutaneous coronary intervention in relation to baseline lesion physiology: results of the JUSTIFY-PCI study. Circ. Cardiovasc. Interv. 2015;8:e01715

20. Wang J, Li S, Chen W, Chen Y, Pang Z, Li J (2021) Diagnostic efficiency of quantification of myocardial blood flow and coronary flow reserve with CZT dynamic SPECT imaging for patients with suspected coronary artery disease: a comparative study with traditional semi-quantitative evaluation. Cardiovasc. Diagn. Ther. 11:56-67

21. Assante R, Mainolfi CG, Zampella E, Gaudieri V, Nappi C, Mannarino $T$ et al (2021) Relation between myocardial blood flow and cardiac events in diabetic patients with suspected coronary artery disease and normal myocardial perfusion imaging. J. Nucl. Cardiol. https://doi.org/10.1007/s12350-021-02533-w

22. Knuuti J, Wijns W, Saraste A, Capodanno D, Barbato E, FunckBrentano C et al (2020) 2019 ESC Guidelines for the diagnosis and management of chronic coronary syndromes. Eur. Heart J. 41:407-477

Publisher's Note Springer Nature remains neutral with regard to jurisdictional claims in published maps and institutional affiliations. 